\section{Parkin western blotting is useful for identification of patients with Parkin-related Parkinson's disease}

Parkinson's disease (PD) is a progressive neurological movement disorder characterised pathologically by degeneration of dopaminergic neurons in the substantia nigra pars compacta and the presence of Lewy bodies. Cardinal clinical features of PD are bradykinesia, rigidity, resting tremor, postural instability and responsiveness to levodopa. Identification of patients with causative variations in the PD-related genes (eg, SNCA, LRRK2, Parkin, PINK1, $D J-1$ and $A T P 13 A 2$ ) has been challenging given that only some patients have distinctive clinical phenotypes or a suggestive family history. Although clinical clues to a specific monogenic form of PD may be observed in some cases, for instance, behavioural problems and postural hypotension in patients with missense mutations in SNCA, early-onset dystonia in patients with mutations in Parkin and vertical gaze palsy, spasticity and facial-faucal mini-myoclonus in patients with mutations in $A T P 13 A 2,{ }^{1} 2$ these manifestations are not universally present. Moreover, patients with mutations in LRRK2 or multiplications in SNCA can be clinically indistinguishable from idiopathic PD. Additionally, variable disease expressivity ${ }^{3}$ and overlapping clinical presentations in the monogenic and idiopathic cases also complicate the diagnostic process to identify each monogenic form of PD. Identification of mutations in Parkin (MIM\# 602544), which are the commonest genetic cause of autosomal recessive PD (MIM\# 600116), requires a combination of direct sequencing and MLPA genetic analysis, given that missense mutations, microdeletions/duplications and large-scale rearrangements and gene dosage effects can all cause Parkin-related PD. ${ }^{4}$

In this study, we determined Parkin expression in PD patient-derived skin fibroblasts, instead of Parkin-deficient blood leuckocytes, to investigate its usability in the diagnosis of Parkin-related PD. Western blotting of Parkin was performed in fibroblasts from individuals with known mutations in PD-related genes (ie, Parkin, PINK1, ATP13A2, and LRRK2), idiopathic $\mathrm{PD}$ and healthy controls (figure 1A-C; detailed description of the samples and method has previously been published $^{23}$ ).

Full-length wild-type Parkin protein $(50 \mathrm{kDa})$ was present in all controls. Consistent with previous findings, ${ }^{6} 7$ the absence of Parkin was observed in the homozygous and compound heterozygous Parkin mutations carriers. The expression of Parkin was significantly decreased in the single heterozygous Parkin mutation carriers to $58 \pm 4.5 \%$ compared to controls. Parkin was present in PINK1, ATP13A2, LRRK2 mutation carriers and in idiopathic PD patients at comparable levels to the healthy controls (figure 1D). These results indicate that Parkin western blotting can be used to efficiently identify Parkin-related PD from other forms of monogenic PD that may similarly present with an autosomal recessive family history, younger age of onset or overlapping phenotype. The possibility of patients harbouring missense mutations in Parkin cannot be excluded entirely by this method since Parkin levels

(a)

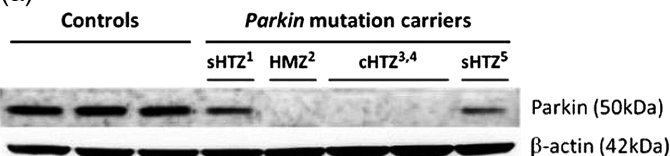

${ }^{1}$ single heterozygous (c.535_871del), ${ }^{2}$ homozygous (c.8_171del) ${ }^{3}$ compound heterozygous (c.8_171del + c.535_871del), ${ }^{4}$ compound heterozygous (c.8_171del + c.337_376del), ${ }^{5}$ single heterozygous (Ex2-4del)

(b)

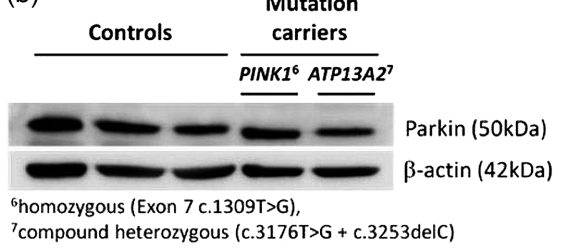

(c)

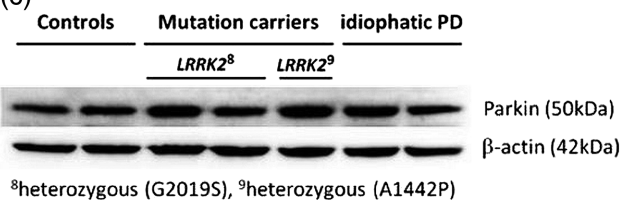

(d)

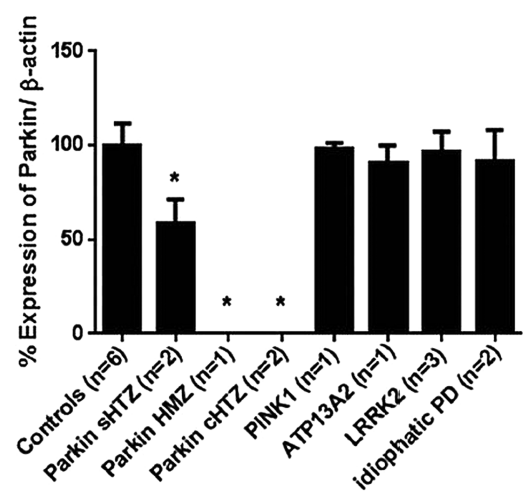

Figure 1 Determination of Parkin protein in human skin fibroblasts. Western blotting using whole-cell lysates from healthy controls, individuals with mutations in Parkinson's disease (PD)-related genes and idiopathic PD. (A) Full-length wild-type Parkin protein ( $\sim 50 \mathrm{kDa})$ was absent in homozygous and compound heterozygous Parkin mutation carriers. A reduced Parkin expression ( 50\%) was observed in the single heterozygote, compared to the healthy controls. The expression of Parkin was comparable between the healthy controls and (B) PINK1, ATP13A2, (C) LRRK2 mutation carriers and idiopathic PD; $\beta$-actin $(42 \mathrm{kDa})$ was used as loading control. (D) Densitometric analysis of the immunoblots. The intensity of each band was normalised to $\beta$-actin and presented as mean $\pm \mathrm{SD}^{*} ; \mathrm{p}<0.001$ in two-tailed Student's $t$ test compared with the healthy controls. 
may not always be altered in this setting. ${ }^{8}$ Additionally, it can be used to confirm the functional deficit on next-generation sequencing protocols when a Parkin mutation has been identified.

In summary, western blotting, to identify Parkin protein expression in skin fibroblast cultures, may be used as a supportive tool to identify patients suspected of having mutations in the Parkin gene and justify further mutational analysis and gene dosage studies for the identification of mutations in Parkin versus other genes that cause monogenic young onset Parkinsonism. Further validation to fully assess the usability of Parkin western blotting in differential diagnosis for Parkinrelated PD using a larger cohort of healthy controls, idiopathic and monogenic PD patients including with mutations in other PD-related genes (eg, SNCA and $D J-1$ ) is warranted.

\section{Brianada Koentjoro, ${ }^{1}$ Jin-Sung Park, Carolyn M Sue ${ }^{1,2}$}

${ }^{1}$ Department of Neurogenetics, Kolling Institute of Medical Research, Royal North Shore Hospital and the University of Sydney, St. Leonards, New South Wales, Australia

${ }^{2}$ Department of Neurology, Royal North Shore Hospital, St. Leonards, New South Wales, Australia

Correspondence to Professor Carolyn M Sue, Department of Neurology, Royal North Shore Hospital and Kolling Institute of Medical Research, University of Sydney, St Leonards, NSW 2065, Australia; carolyn.sue@sydney.edu.au

Acknowledgements The authors thank the patients for participating in this project.

Contributors BK: drafting/revising the manuscript, study concept or design, analysis or interpretation of data, acquisition of data. Dr J-SP: drafting/revising the manuscript, study concept or design, analysis or interpretation of data, study supervision or coordination. Dr CMS: drafting/revising the manuscript, study concept or design, analysis or interpretation of data, study supervision or coordination, obtaining funding.

Funding This work was supported by grants from the Australian Brain Foundation and the Parkinson's New South Wales Association.

Competing interests CMS has been awarded an NHMRC Clinical Practitioner Fellowship and is supported by the Ramsay Health Teaching and Research Fund.

Ethics approval Northern Sydney \& Central Coast Health Human Research Ethics Committee.

Provenance and peer review Not commissioned; externally peer reviewed.

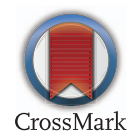

To cite Koentjoro B, Park J-S, Sue C M. J Neurol Neurosurg Psychiatry 2014;85:1436-1437.

Received 19 March 2014

Accepted 24 March 2014

Published Online First 9 May 2014
J Neurol Neurosurg Psychiatry 2014;85:1436-1437. doi:10.1136/jnnp-2014-308142

\section{REFERENCES}

1 Klein C, Lohmann-Hedrich K. Impact of recent genetic findings in Parkinson's disease. Curr Opin Neurol 2007:20:453-64.

2 Park JS, Mehta P, Cooper AA, et al. Pathogenic effects of novel mutations in the P-type ATPase ATP13A2 (PARK9) causing Kufor-Rakeb syndrome, a form of early-onset parkinsonism. Hum Mutat 2011;32:956-64.

3 Koentjoro B, Park JS, Ha AD, et al. Phenotypic variability of parkin mutations in single kindred. Mov Disord 2012;27:1299-303.

4 Hedrich K, Eskelson C, Wilmot B, et al. Distribution, type, and origin of Parkin mutations: review and case studies. Mov Disord 2004;19:1146-57.

5 Huang $Y$, Halliday GM, Vandebona $\mathrm{H}$, et al. Prevalence and clinical features of common LRRK2 mutations in Australians with Parkinson's disease. Mov Disord 2007;22:982-9.

6 Rakovic A, Grunewald A, Seibler P, et al. Effect of endogenous mutant and wild-type PINK1 on Parkin in fibroblasts from Parkinson disease patients. Hum Mol Genet 2010;19:3124-37.

7 Pacelli C, De Rasmo D, Signorile A, et al. Mitochondrial defect and PGC-1alpha dysfunction in parkin-associated familial Parkinson's disease. Biochim Biophys Acta 2011;1812:1041-53.

8 Gu WJ, Corti O, Araujo F, et al. The C289G and C418R missense mutations cause rapid sequestration of human Parkin into insoluble aggregates. Neurobiol Dis 2003;14:357-64. 